\title{
Goodbye to brute force in antibiotic discovery?
}

\author{
Roberto Kolter and Gilles P. van Wezel
}

\author{
Widespread antibiotic resistance is a growing public health problem. Can we revive large-scale \\ screening to keep the pipelines flowing or will we depend increasingly on biological and \\ ecological insights?
}

T he discovery and development of antibiotics to fight bacterial diseases is one of the great triumphs in modern medicine. However, the rapid spread of resistance to antibiotics, recognized as a major limitation ever since their introduction, means that bacterial infections continue to threaten world health. The number of human deaths worldwide due to antimicrobial resistance is expected to rise from 700,000 per year today to over 10 million by $2050^{1}$. There is a real danger that the era of antibiotics might end a mere century after their first clinical use. We argue that to prevent this from happening, those working in antimicrobial discovery should consider a combination of harnessing new biodiversity, ecological understanding of the triggers and cues that activate laboratorysilent biosynthetic gene clusters, and nextgeneration high-throughput screening, consisting of genome sequencing and synthetic biology.

The reasons for the increase in the frequency of antibiotic resistance are complicated (including overuse, abuse and lack of enforceable regulatory controls) but as a result we are now running out of useful antibiotics. Indeed, antibiotic resistance is an evolutionary inevitability and thus new antibiotics will always be needed. However, the rate of new antibiotics entering clinical use has come to a grinding halt in recent decades. Why is this so? For one, the path that pharmaceutical companies need to take to bring a lead compound to the clinic is long and extremely costly and the eventual payback is relatively low, with the risk that new drugs will be kept on the shelf as a last line of defence. A second problem is replication, that is, searches for new antibiotics tend to yield antibiotics that are already known. Addressing the high frequency of replication requires innovative strategies to replenish antimicrobial drug pipelines ${ }^{2,3}$.

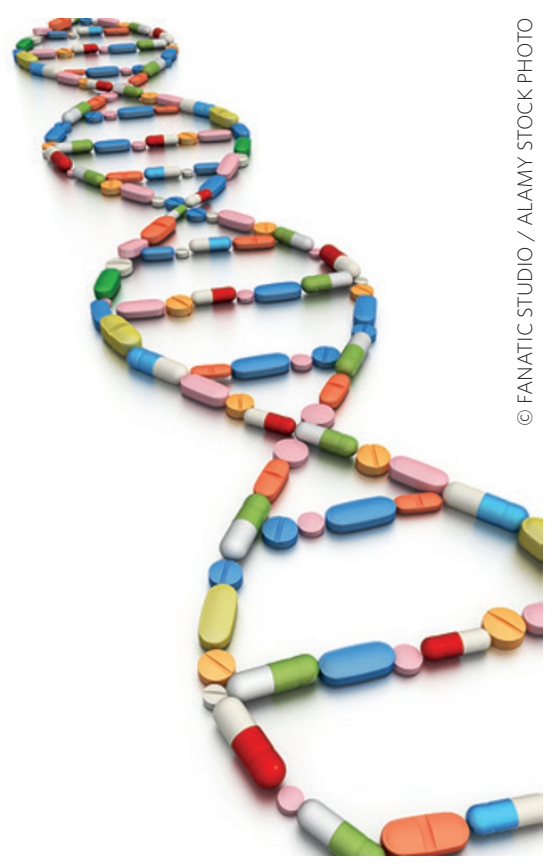

From the early days of microbiology, and classically represented in Alexander Flemming's discovery of penicillin, antibiotic activities have initially been detected as growth inhibition zones on lawns of sensitive bacteria around where an antibiotic producer is growing. This screening approach was developed into a more elaborate platform by Selman Waksman, leading to the discovery of the anti-tuberculosis drug streptomycin. This 'brute force' approach was used throughout the world to screen countless microbial isolates for antibiotic production. And it was very successful too, leading to the so-called golden era of antibiotic discovery. During the 1950s and 1960s, thousands of compounds with antibiotic activity were discovered and many were developed for clinical use. Initial efforts were so intensive that by the 1970s the rate of new compound discovery slowed down dramatically. The 'low hanging fruit' had been harvested and it seemed that all compounds with antibiotic activity had been discovered. Development of synthetic antibiotics was subsequently attempted but these largely met with failure. Combinatorial libraries proved to cover relatively small regions of chemical space. Designing drugs to inhibit specific targets also failed because many antibiotics do not obey Lipinski's rule of five (making chemical synthesis less predictable) or because compounds do not pass the bacterial cell envelope $e^{4}$. Therefore, natural products, resulting from millions of years of evolution, still remain the best starting point for antibiotic development. Yet, by the end of the twentieth century all indications were that all extant antibiotics had been found. Not because those working in drug discovery had made mistakes, but ironically - because they had applied the brute force approach too well. We propose that the future direction for antibiotic discovery now lies elsewhere, in following less-trodden paths.

Two developments show that the surface had barely been scratched in terms of discovering new compounds with antibiotic activity. First came the recognition that after a century of developing cultivation techniques, we are still unable to cultivate the vast majority of environmental bacteria. Referred to as the 'great plate count anomaly', for every 100 bacteria seen under the microscope, only one would grow. Given that industrial screening for antibiotics had involved pure cultures of microorganisms, it is clear that only a small fraction of the microbial world had been surveyed for antibiotic production. Second, it also became evident that even in those microorganisms that were amenable to cultivation, many compounds were not produced under routine screening conditions. The genomes of Streptomyces avermitilis ${ }^{5}$ and S. coelicolor ${ }^{6}$, 
which had been studied by thousands of scientists for decades, revealed many gene clusters that should specify antibioticlike substances, and this is true for most actinomycete genomes ${ }^{7}$. Yet no products from those gene clusters had been identified. It is likely that, rather than the gene clusters being always silent, we simply do not have sufficient insights into the biology and ecology of antibiotic production to understand the triggers and cues required to activate their expression. Once obtained, such understanding may be translated to find elicitor molecules or conditions to facilitate screening ${ }^{8}$. These developments suggest that replacing brute force with better-informed 'clever' approaches should lead the way.

To exploit the 'not-yet-cultivated' potential of the microbial world, we are learning how to cultivate this unexplored biodiversity, and this has led to some promising results. One such approach has been to cultivate microorganisms in the soil they originate from, ensuring the supply of all necessary nutrients and trace elements, which led to the discovery of a peptide antibiotic produced by a novel species ${ }^{9}$. Another approach aims to harness microbial biodiversity by targeting bacteria from taxa that have not been studied extensively and/ or have been isolated from complex or remote ecological sites including the deep ocean, deserts, high mountains as well as plant endophytes or animal symbionts. However, it remains to be seen if the yet unexplored biodiversity will be sufficiently different from the already characterized taxa in terms of biosynthetic potential to yield clinically useful antibiotics.

In traditional cultivation, bacteria were grown in isolation, inevitably missing out on many of the signals that trigger the production of natural products in the original habitat. Realizing that bacteria produce new compounds when in the presence of other bacterial or eukaryotic microorganisms, co-cultivation approaches have been adopted and have revealed a very different universe of secreted natural products than that observed from pure cultures $^{10-12}$. We believe that this is an approach with very strong potential, especially because this line of screening has not been followed in the past.

Whether the 'hits' that have so far been, and are likely to continue to be, identified as a result of these 'clever' approaches will eventually reach the clinic still remains to be seen. So, what else can be done to keep the pipelines of novel lead compounds flowing? We expect that there will soon be the opportunity to once again apply a brute force approach. Recent advances in high-throughput genome sequencing and bioinformatic analyses now make it feasible to carry out an ongoing census of the extant microbial genes and gene clusters potentially specifying new antibiotics, without the need for cultivation ${ }^{13,14}$. Still, the same rules apply here; many of the identified gene clusters will remain silent, and the potential application of synthetic biology approaches to express these clusters and harvest their products are still low-throughput and expensive. However, it is likely that as was seen for DNA sequencing in recent years, the process of DNA synthesis will increase in speed and dramatically drop in cost in the near future. This will make it feasible to synthesize, clone and express thousands or even millions of biosynthetic gene clusters in heterologous hosts followed by assays for antibiotic activity. This might appear far-fetched but it really is not. If a drive similar to the human genome sequencing project could fuel gene synthesis efforts, a situation may arise where literally millions of novel gene clusters could become accessible. Looking back at the history of science, this moment may be closer than anyone can presently foresee. We believe that by focusing on both the clever biological and ecological approaches as well as on genomics- and synthetic-biologybased brute force, scientists will discover enough lead compounds to keep supplying mankind with the new antimicrobials that are so greatly needed.

Roberto Kolter is in the Department of Microbiology and Immunobiology, Harvard Medical School, 77 Avenue Louis Pasteur, Boston, Massachusetts 02115, USA. Gilles P. van Wezel is in the Department of Molecular Biotechnology, Institute of Biology, Leiden University, Sylviusweg 72, 2333 BE Leiden, The Netherlands, and the Department of Microbial Ecology, Netherlands Institute for Ecology (NIOO-KNAW), Droevendaalsesteeg 10, 6708 PB Wageningen, The Netherlands.

e-mail:kolter@hms.harvard.edu; g.wezel@biology.leidenuniv.nl

\section{References}

1. Antimicrobial Resistance: Tackling a Crisis for the Health and Wealth of Nations (Review on Antimicrobial Resistance, 2014).

2. Cooper, M. A. \& Shlaes, D. Nature 472, 32 (2011).

3. Payne, D. J., Gwynn, M. N., Holmes, D. J. \& Pompliano, D. L. Nature Rev. Drug Discov. 6, 29-40 (2007).

4. Lewis, K. Nature Rev. Drug Discov. 12, 371-387 (2013).

5. Ikeda, H. et al. Nature Biotechnol. 21, 526-531 (2003).

6. Bentley, S. D. et al. Nature 417, 141-147 (2002).

7. Nett, M., Ikeda, H. \& Moore, B. S. Nat. Prod. Rep. 26, 1362-1384 (2009)

8. Rigali, S. et al. EMBO Rep. 9, 670-675 (2008).

9. Ling, L. L. et al. Nature 517, 455-459 (2015).

10. Schroeckh, V. et al. Proc. Natl Acad. Sci. USA 106, 14558-14563 (2009).

11. Traxler, M. F., Watrous, J. D., Alexandrov, T., Dorrestein, P. C. \& Kolter, R. mBio 4, (2013).

12. Wilson, M. C. et al. Nature 506, 58-62 (2014).

13. Cimermancic, P. et al. Cell 158, 412-421 (2014).

14. Doroghazi, J. R. et al. Nature Chem. Biol. 10, 963-968 (2014). 